\title{
Orthogonal Analysis of the Weights based on the Factors Influencing the Total Time of Community Opening
}

\author{
Zengji Wang \\ School of North China Electric Power University, Baoding 071003, China; \\ 1379130580@qq.com
}

Keywords: community opening; orthogonal analysis; factors' weight; Dijkstra algorithm

\begin{abstract}
According to the urban planning and construction, open community becomes more and more popular in recent years. However, the study of the impact of people on the travel after the opening of the community is not deep. Moreover, the impact of each factor on the degree of travel is not the same. Therefore, this paper takes the total travel time of the crowd as the total index and the free flow time in the district, the influence coefficient of the internal road delay, the free flow time of the external roads, the influence coefficient of the external road delay, the intersection delay time as the five influencing factors of the index. Then we use Dijkstra algorithm getting the simulation data and carry out two levels of seven factors orthogonal test. Later we use the range analysis method to get the weight. Furthermore, the influence degree of each factor on travel time is obtained.
\end{abstract}

\section{Introduction}

According to the national policy, in principle, a closed residential area won't be built. Residential quarters and units compound which have been completed should be gradually open. However, the focuses of the discussion is whether the opening communities can achieve the objective of optimizing the road network structure, improving the road capacity, improving the traffic conditions and improving the effectiveness.

Orthogonal experimental design is a multi-factor and multi-level design method, which is based on orthogonality from the comprehensive test to select some representative points to test. ${ }^{[1]}$ Range analysis can reflect the influence degree of each factor. This can provide a reasonable basis for the opening community.

Dijkstra algorithm is a typical single source shortest path algorithm for computing a node to all other nodes of the shortest path. The main feature is to start at the center of the layers of expansion until the extension to the end. ${ }^{[2]}$

\section{Symbols and Definitions}

Table 1 variable description

\begin{tabular}{cc}
\hline Abbreviation & Meaning \\
\hline TTT & the total travel time of the crowd \\
FFTI & the free flow time in the district \\
ICI & the influence coefficient of the internal road delay \\
FFTE & the free flow time of the external roads \\
ICE & the influence coefficient of the external road delay \\
IDT & the intersection delay time \\
\hline
\end{tabular}




\section{The research process of the weight of each factor}

In this section, we take the total time of travel as the index, and identify five factors according to the actual situation. After that, we use graph theory to get the simulation data, and carry on the orthogonal experiment. Then the weight of each factor is obtained after using the range analysis.

\subsection{The reason for the selection of indicators}

Because the basic function of the road network is to meet the most people with the least time to reach the best destination. ${ }^{[3]}$ If the community is open, the total travel time of the crowd reduced. This indicates that the opening community improves the traffic situation, on the contrary, there is no improvement.

Obviously, FFTI, ICI, FFTE, ICE, IDT can have a great significant impact on the total travel time of the crowd. So, it is very reasonable for us to choose these five indicators.

\subsection{Orthogonal experimental design}

\subsubsection{Acquire the simulation data}

First, we simplify the district into a map. Then we use the Dijkstra algorithm to calculate the minimum path from the starting point to the destination and calculate the sum of the travel time of all the people. The simulation data of the orthogonal experiment are obtained.

\subsubsection{Proper orthogonal table}

Because the orthogonal experiment has five factors and two levels, we choose $L_{8}\left(2^{7}\right)$.

Table 2 Orthogonal table- $L_{8}\left(2^{7}\right)$

\begin{tabular}{|c|c|c|c|c|c|c|c|}
\hline $\begin{array}{c}\text { Test } \\
\text { sequenc }\end{array}$ & a & b & c & $d$ & $e$ & $f$ & $g$ \\
\hline $\mathbf{e}$ & & & & & & & \\
\hline 1 & 1 & 1 & 1 & 1 & 1 & 1 & 1 \\
\hline $\mathbf{2}$ & 1 & 1 & 1 & 2 & 2 & 2 & 2 \\
\hline 3 & 1 & 2 & 2 & 1 & 1 & 2 & 2 \\
\hline $\mathbf{4}$ & 1 & 2 & 2 & 2 & 2 & 1 & 1 \\
\hline $\mathbf{5}$ & 2 & 1 & 2 & 1 & 2 & 1 & 2 \\
\hline $\mathbf{6}$ & 2 & 1 & 2 & 2 & 1 & 2 & 1 \\
\hline $\mathbf{7}$ & 2 & 2 & 1 & 1 & 2 & 2 & 1 \\
\hline $\mathbf{8}$ & 2 & 2 & 1 & 2 & 1 & 1 & 2 \\
\hline
\end{tabular}

Note: F and G are empty columns.

Table 3 Factors before and after opening

\begin{tabular}{ccc}
\hline Metric & Before & After \\
\hline ICI & $70 \%$ & $100 \%$ \\
FFTE & $70 \%$ & $100 \%$ \\
ICE & $70 \%$ & $100 \%$ \\
IDT & $70 \%$ & $100 \%$ \\
FFTI & $70 \%$ & $100 \%$ \\
\hline
\end{tabular}




\subsubsection{Orthogonal experiment ${ }^{[4]-[6]}$}

Firstly, the data obtained from the above simulation should be brought into the orthogonal table $L_{8}\left(2^{7}\right)$.Secondly, at the same level, we calculate the sum of the travel time corresponding to the five indexes, which are recorded as $\mathrm{K} 1, \mathrm{~K} 2$, and put them under the orthogonal table. Then we subtract the five factors of the corresponding $\mathrm{K} 1, \mathrm{~K} 2$, and determine the order of the five factors by the magnitude of the range.

Table 4 A case study of Changsha City, Hunan Province,China

\begin{tabular}{|c|c|c|c|c|c|c|c|c|}
\hline $\begin{array}{c}\text { Test } \\
\text { sequenc } \\
\mathbf{e}\end{array}$ & \multicolumn{7}{|c|}{ Factors } \\
\hline $\mathbf{1}$ & 1 & 1 & 1 & 1 & 1 & 1 & 1 & 420300 \\
\hline $\mathbf{2}$ & 1 & 1 & 1 & 2 & 2 & 2 & 2 & 620020 \\
\hline $\mathbf{3}$ & 1 & 2 & 2 & 1 & 1 & 2 & 2 & 579480 \\
\hline $\mathbf{4}$ & 1 & 2 & 2 & 2 & 2 & 1 & 1 & 967620 \\
\hline $\mathbf{5}$ & 2 & 1 & 2 & 1 & 2 & 1 & 2 & 638210 \\
\hline $\mathbf{6}$ & 2 & 1 & 2 & 2 & 1 & 2 & 1 & 840640 \\
\hline $\mathbf{7}$ & 2 & 2 & 1 & 1 & 2 & 2 & 1 & 640980 \\
\hline $\mathbf{8}$ & 2 & 2 & 1 & 2 & 1 & 1 & 2 & 691390 \\
\hline K1 & 2587420 & 2519170 & 2372690 & 2278970 & 2531810 & & & \\
\hline K2 & 2811220 & 2879470 & 3025950 & 3119670 & 2866830 & & & \\
\hline $\mathbf{R}$ & 223800 & 360300 & 653260 & 840700 & 335020 & & & \\
\hline Weights & $9.27 \%$ & $14.93 \%$ & $27.07 \%$ & $34.84 \%$ & $13.88 \%$ & & & \\
\hline
\end{tabular}

\subsection{Result analysis}

The weights of the five factors can be seen as follows: IDT $>$ ICE $>$ FFTE $>$ FFIT $>$ ICI. And the first two factors account for $61.91 \%$, while the remaining account for about $38.09 \%$. This indicates that the change of IDT and ICE in the opening community have a great influence on road traffic. This is in line with the actual situation. So the weights are reasonable.

\section{Summary}

Based on Orthogonal experiment, the paper has been studied the Weights based on the Factors Influencing the Total Time of Community Opening. On the above, we take a district as an example. The weights of five factors are obtained by orthogonal experiment. And we find them in conformity with the actual situations. So, it can help us to understand which factor affects the most on traffic after a district is opening. Then government planning departments can try to change the factors to improve traffic capacity. This will be very practical. Of course, this method should be applied according to local conditions, to determine the final results reliably.

\section{References}

[1] http://baike.so.com/doc/5356113-5591604.html

[2] http://baike.so.com/doc/6369076-6582719.html 
[3] Ye Pengyao, Chen Xiaohong. Study on the optimal road network density based on traffic efficiency [J]. China Journal of highway and transport, 2008, 21 (): 94-98.

[4] Zhou Xinyu, Wu Zhijian, Wang Mingwen. Artificial bee colony orthogonal experimental design of [J]. software based on the algorithm, 2015, (09): 2167-2190.

[5] Wang Kan, Yu Jiahua. Analysis of influence of variable width disc shear parameters on shear force based on Deform simulation and orthogonal test method [J]. mechanical and electrical information, 2013, (): 144-145+147.

[6] Han Zhiyan, Wang Jian, Optimization of feature parameters for speech recognition based on orthogonal experimental design [J]. Chinese Journal of computer science, 2010, (): $214-216+250$. 